\title{
Nonrandom spatial clustering of spontaneous anterior fossa cerebrospinal fluid fistulas and predilection for the posterior cribriform plate
}

\author{
Richard D. Murray, MD, ${ }^{1}$ Rachel Friedlander, AB, ${ }^{1}$ Samuel Hanz, ${ }^{1}$ Harminder Singh, MD, ${ }^{1}$ \\ Vijay K. Anand, MD, ${ }^{2}$ and Theodore H. Schwartz, MD ${ }^{1-3}$
} Departments of ${ }^{1}$ Neurosurgery, ${ }^{2}$ Otolaryngology, and ${ }^{3}$ Neuroscience, Weill Cornell Medical College, NewYork-Presbyterian
Hospital, New York, New York

\begin{abstract}
OBJECTIVE The anterior skull base is a common site for the spontaneous development of meningoceles, encephaloceles, and meningoencephaloceles that can lead to cerebrospinal fluid (CSF) fistula formation, particularly in association with idiopathic intracranial hypertension. In some circumstances the lesions are difficult to localize. Whether all sites in the anterior skull base are equally prone to fistula formation or whether they are distributed randomly throughout the anterior skull base is unknown, although the anterior cribriform plate has been proposed as the most frequent location. The purpose of this study was to identify sites of predilection in order to provide assistance for clinicians in finding occult leaks and increase the understanding of the etiology of this pathology.
\end{abstract}

METHODS The authors performed a retrospective review of a prospectively acquired surgical database of all endonasal endoscopic surgeries performed at Weill Cornell Medical College by the senior authors. Spontaneous CSF fistulas of the anterior skull base were identified. The anatomical sites of the defects were located on radiographic images and normalized to a theoretical $4 \times 2$ grid representing the anterior midline skull base. Data from the left and right skull base were combined to increase statistical power. This grid was then used to analyze the distribution of defects. Frequency analysis was performed by means of a chi-square test, with a subsequent Monte Carlo simulation to further strengthen the statistical support of the conclusions.

RESULTS Nineteen cases of spontaneous CSF fistulas were identified. Frequency analysis using chi-square indicated a nonrandom distribution of sites $(p=0.035)$. Monte Carlo simulation supported this conclusion $(p=0.034)$. Seventy-four percent of cases occurred in the cribriform plate $(p=0.086)$. Moreover, $37 \%$ of all defects occurred in the posterior third of the cribriform plate.

CONCLUSIONS Anterior skull base spontaneous CSF leaks are distributed in a nonrandom fashion. The most likely site of origin of the spontaneous CSF leaks of the anterior midline skull base is the cribriform plate, particularly the posterior third of the plate, likely because of the lack of significant thick bony buttressing. Clinicians searching for occult spontaneous leaks of the anterior skull base should examine the cribriform plate, especially the posterior third with particularly close scrutiny.

https://thejns.org/doi/abs/10.3171/2016.4.JNS152975

KEY WORDS endoscopic endonasal cerebrospinal fluid fistula repair; endonasal encephalocele; endonasal meningocele; endonasal meningoencephalocele; cribriform plate encephalocele; pituitary surgery

EREBROSPINAL fluid (CSF) fistulas can be acquired or congenital. Acquired fistulas can be traumatic or spontaneous. The etiology of spontaneous CSF fistulas often involves a combination of chronic increases in intracranial pressure (ICP), either from idiopathic intracranial hypertension (IIH) or obesity and an anatomical predisposition or thinning of the cranial base adjacent to an area of pneumatization. ${ }^{2,4,17}$ Spontaneous CSF leaks involve a herniation of the meninges either without or with brain tissue, labeled as meningoceles or meningoencephaloceles, respectively. The most common site of spontaneous CSF leaks is the anterior cranial fossa, which consists

ABBREVIATIONS BMI = body mass index; CSF = cerebrospinal fluid; ICP = intracranial pressure; $I \mathrm{IH}=$ idiopathic intracranial hypertension.

SUBMITTED December 20, 2015. ACCEPTED April 29, 2016.

INCLUDE WHEN CITING Published online July 1, 2016; DOI: 10.3171/2016.4.JNS152975. 
of the fovea ethmoidalis (ethmoid roof) and the cribriform plate. Previous authors have proposed the ethmoid roof to be the area of most frequent spontaneous CSF leaks. ${ }^{2}$ However, the bone of the cribriform plate is extremely thin, and multiple ostia and dural and arachnoid sleeves penetrate the bone to permit the passage of the olfactory epithelia. ${ }^{6}$

CSF fistulas require repair because communication between the nasal passage and intracranial space can lead to meningitis. Although a variety of approaches have been used in the past, most surgeons now perform endonasal surgery for the majority of anterior skull base CSF fistulas with a success rate greater than $90 \%{ }^{4,12,14}$ However, spontaneous CSF leaks can sometimes be difficult to find on preoperative imaging and often require invasive testing such as CT cisternography and even intraoperative examination with intrathecal fluorescein. ${ }^{2,4}$ A map of the distribution of common sites of spontaneous CSF fistulas would be useful for clinicians to guide such skull base examinations.

Given the importance of the underlying anatomical weakness of the skull base in the formation of spontaneous CSF fistulas, the anatomy of the cribriform plate has been examined to assess sites of possible fistula predilection. In 1962, Keros described 3 variations in the relationship between the lamina cribrosa and the lateral lamella to demonstrate how iatrogenic injuries may form in congenitally low-lying plates. ${ }^{11}$ Other authors have proposed the posterior ethmoid as the most common site of iatrogenic injury. ${ }^{20}$ Spontaneous CSF leaks, on the other hand, have been associated with anterior cribriform plate and anterior fovea ethmoidalis, because of the location of the ethmoid foramen and cribroethmoidal foramen, respectively. ${ }^{10,16}$ However, no study has examined the actual distribution of anterior cranial fossa spontaneous CSF leaks to determine whether they are randomly distributed or cluster in specific anatomical locations. In this study we found that the posterior third of the cribriform plate is the weakest area of the anterior skull base, and we propose a simple hypothesis to explain our findings.

\section{Methods}

We retrospectively queried a prospectively acquired database of all endonasal endoscopic surgeries done at Weill Cornell Medical College, NewYork-Presbyterian Hospital for all patients older than 16 years who underwent endoscopic endonasal surgery for the treatment of anterior cranial base spontaneous CSF fistula, encephalocele, meningocele, or meningoencephalocele during the period of January 2004 to April 2015. Patients with congenital, traumatic, iatrogenic (previous skull base or sinonasal surgery), infectious, or tumoral causes of CSF leak were excluded from this study. Reoperations and cases with unavailable or inadequate preoperative imaging were also excluded.

Clinical records were reviewed and the following data points collected: age, sex, possible prior cause, body mass index (BMI), and a diagnosis of pseudotumor cerebri. Operative reports were reviewed, and the locations identified on these reports were compared with sites of leakage encountered during surgery. Clinical data were then statistically analyzed.
The preoperative radiographic images obtained in all patients were reviewed, and measurements were made of the precise size and location of the defect in the anterior cranial fossa leading to the encephalocele or meningocele or meningoencephalocele.

To allow for direct comparison between different cases as well as statistical analysis, individual patient measurements were normalized to a hypothetical standard anterior skull base with a square geometry measuring $40 \mathrm{~mm}$ per side (Fig. 1).

The normalization process involved the following steps.

For lesions involving the cribriform plate, a factor was derived that would convert the anteroposterior length of the cribriform plate to the desired length of $30 \mathrm{~mm}$. This same factor was then applied to the anteroposterior location and anteroposterior length of the osteodural defect. For lesions in the anterior third of the cribriform plate, a factor was derived that would convert the anterior cribriform plate width to the required $10-\mathrm{mm}$ measurement. This factor was then applied to the mediolateral location and width of the osteodural defect measurements. For lesions in the middle and posterior third of the cribriform plate, the widest and most posterior diameters were used in the same method to calculate the appropriate factor.

For lesions involving the ethmoid roof, the normalization process involved trigonometric evaluation of the an-

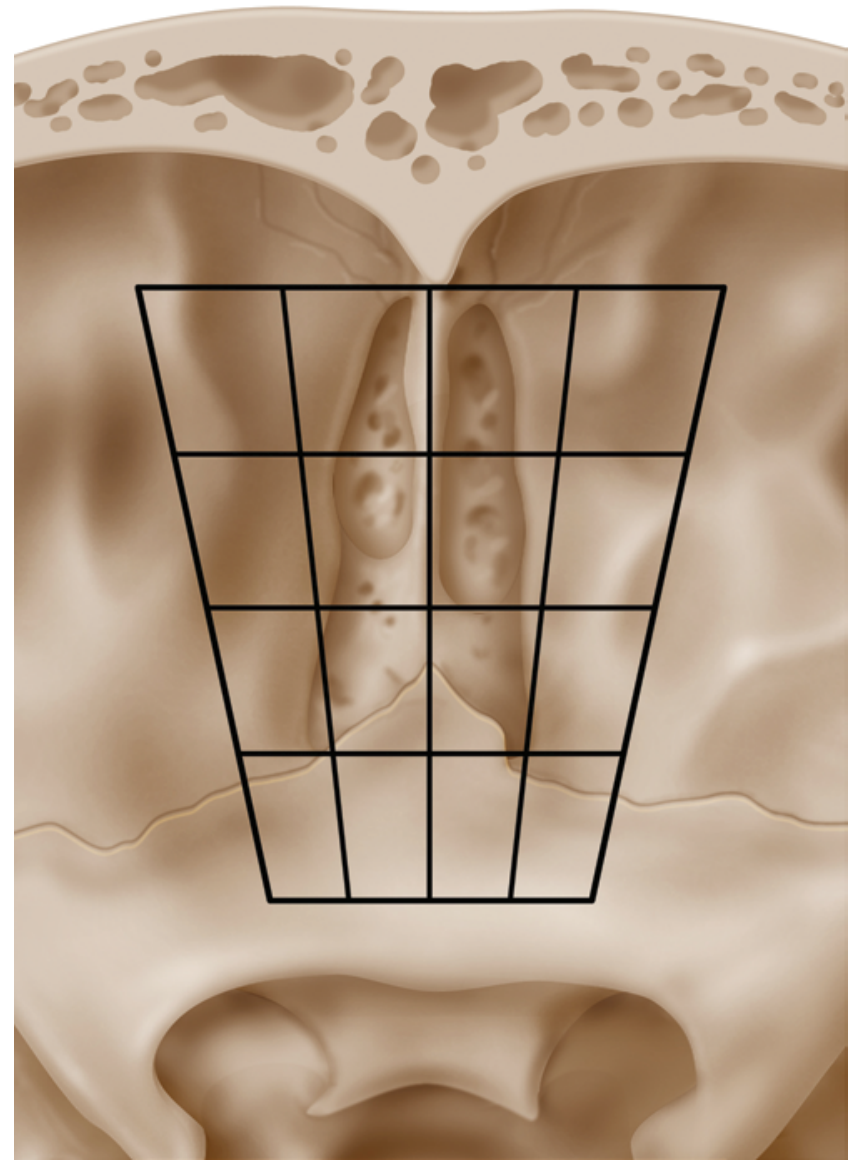

FIG. 1. Normalized anterior skull base grid divided into 16 locations with respect to the cribriform plate. Figure is available in color online only. 


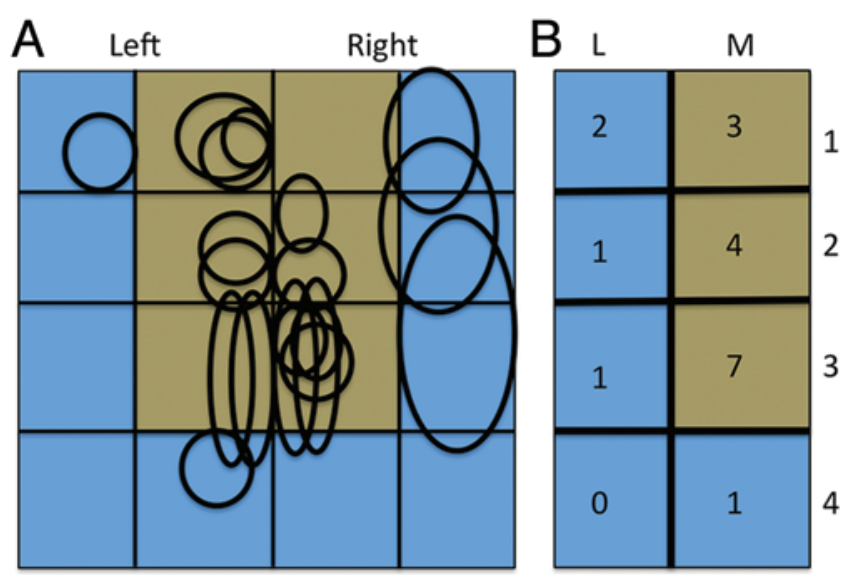

FIG. 2. Graphical and numeric representation of the distribution of lesions for the entire grid $(\mathbf{A})$ and the combined left and right grid (B) superimposed on each other. Figure is available in color online only.

teroposterior location of the lesion in relation to the hypothetical trapezoid formed by the ethmoid roof. This then determined the factor required to calculate the theoretical lateral displacement of the lesion as well as the mediolateral dimensions. Thereafter the anteroposterior location was normalized to a 40-mm length.

Once the normalization process was completed, a theoretical $40 \times 40-\mathrm{mm}$ square was constructed and all lesions were superimposed on this for visual reference (Figs. 1 and 2).

Since the anterior skull base is symmetrical and there is no a priori reason to think that the left and right anterior skull base would have unique predispositions to meningoceles in a given location, we combined the data from the left and right sides to increase statistical power. Although previous research has shown that the cribriform plate dimensions may vary widely between left and right in a single individual, when multiple individuals are compared with one another, this variation tends to cancel itself out, and the average dimensions of the left cribriform plate are very similar to those of the right cribriform plate. ${ }^{8}$ This allowed the superimposition of the left- and right-sided defects in our study, so that a single grid of $20 \times 40 \mathrm{~mm}$ was then created, which represented both left- and right-sided lesions, while maintaining their mediolateral orientation (Fig. 2).

Thereafter this grid was divided into 8 squares, with squares M1-3 representing the anterior, middle, and posterior cribriform plate and L1-3 representing the ethmoid roof, or fovea ethmoidalis, lateral to the cribriform plate. Lastly, M4 and L4 represented the ethmoid roof posterior to the cribriform plate on the medial and lateral aspects, respectively. The epicenter of each defect was then used to determine into which position on the grid each defect would be categorized (Fig. 2). For statistical analysis, the final dataset consisted of 8 possible sites into which each defect was positioned (Fig. 2).

Statistical analysis utilizing frequency analysis was subsequently performed. First, a goodness-of-fit test was performed to determine whether the number of cases per site was randomly distributed. Because the chi-square test should ideally be performed with more data, the test was also performed with 5000 Monte Carlo simulations.

\section{Results}

Nineteen patients fulfilled the inclusion and exclusion criteria. Of these, 15 (79\%) were female. The patients' mean age was 42 years (range 16-66 years). The average preoperative BMI for which the data were available was 30.4 (range 21.4-39; 14 patients). In 12 patients the BMI was greater than 25, and in 9 of these greater than 30 .

Empty sella syndrome was present in 11 cases (58\%). Operative reports were carefully scrutinized to determine the intraoperative site of leakage. In all available cases the operative report stated that stereotactic neuronavigation was used to localize the defect. In 13 cases it was possible to deduce from the operative report the exact lesion location. In 2 cases no operative report was available, and in the other 4 cases the wording used was too vague to accurately determine the site.

Symptoms and signs included headache (in all 19 cases), CSF rhinorrhea (in 17), prior episodes of meningitis (in 7), and various other nonspecific complaints, including recurrent sinusitis, photophobia, and malaise.

Of note, none of these patients had a history of significant head injury. One 26-year-old male patient with a BMI of 21 had recently undergone intraventricular surgery for a colloid cyst, after which he had an external ventricular drain for a few days.

Radiographic analysis yielded 10 right-sided and 9 leftsided lesions, with 14 lesions being located in the cribriform plate and 5 in the ethmoid roof or fovea ethmoidalis (Fig. 2).

\section{Statistical Analysis}

A goodness-of-fit test was performed to determine whether the number of cases per site was randomly distributed. Accordingly, the chi-square test yielded a p value of 0.035 . This indicates a nonrandom distribution of leakage sites. We also performed a Monte Carlo simulation in which 5000 random permutations of stochastically distributed leakage sites were compared with the actual data. This yielded a $\mathrm{p}$ value of 0.034 , further confirming a nonrandom distribution of sites. The null hypothesis of uniform distribution of cases over the sites was thus rejected at a 5\% significance level.

The majority of leaks (74\%) occurred in the cribriform plate (M1, M2, and M3) ( $\mathrm{p}=0.0086$ for comparison with all other sites, Fisher's exact test). Moreover, 37\% of all leaks $(n=7)$ occurred in the posterior compartment of the cribriform plate (M3). A subsequent subset analysis was performed comparing the distribution of leaks within the cribriform plate (M1, M2, and M3). Although 50\% ( $\mathrm{n}=$ 7) of the leaks occurred within the posterior third of the cribriform plate, there were not enough samples for statistical significance.

\section{Discussion}

Spontaneous CSF leaks arise from a combination of a constant or recurrent increase in ICP and a structural 
weakness of the skull base. Roughly $70 \%$ of patients with spontaneous CSF fistulas have evidence of IIH and the majority are obese and female. ${ }^{2,4}$ If the fistula is left untreated, the risk of meningitis is roughly $19 \%-25 \%{ }^{7,13} \mathrm{El}-$ evated ICP creates a fistula that acts as a release valve to lower ICP, which can then become elevated again once the defect is repaired..$^{22,23}$ The development of increased ICP involves a complex interaction between a variety of predisposing factors. Obesity causes a reduction in venous return and an increase in ICP which can lead to thinning of the bones of the skull base, compression of the arachnoid granulations, and pressure on the venous sinuses. ${ }^{4}$ IIH can lead to empty sella, which may impair the hypothalamicpituitary axis and cause obesity., ${ }^{5,21}$ The unique hormone fluctuations of the female sex and their manipulation with oral contraceptives can alter coagulation, leading to sinus thrombosis and IIH.

The purpose of this paper, however, was to examine the other half of the equation-namely, the etiology of the congenital or acquired weakness in the skull base that leads to the formation of a CSF fistula in a specific location. The first question that arises is whether CSF fistulas are distributed randomly throughout the anterior fossa or in a patterned fashion. Our data clearly show a nonrandom distribution with more frequent leaks in the cribriform plate than in the fovea ethmoidalis. Moreover, the leaks were much more frequent in the posterior third of the cribriform plate, which is actually contrary to what has been previously theorized.

It may not be surprising that the dural dehiscences and arachnoid sleeves that accompany the olfactory fila to the olfactory recess are predisposing factors in the etiology of CSF fistulas. ${ }^{6}$ However, previous authors have proposed the anterior ethmoid roof as the weakest area of the anterior fossa as a result of the passage of the anterior ethmoidal artery through the anterior ethmoidal foramen. ${ }^{10} \mathrm{~A}$ second foramen, named the "ethmoid foramen" was also proposed as the weakest area of the skull base. Originally described by Rouvière in 1911, this foramen is adjacent to the crista galli and was recently demonstrated to be as large as $6.5 \mathrm{~mm}$ and theorized to permit the passage of the " 0 " or " $\mathrm{N}$ " cranial nerve carrying autonomic/neuromodulatory as well as sensory neurons that release luteinizing hormone-releasing hormone. ${ }^{16,19}$ Our data directly contradict these prior theories because the anterior ethmoid and anterior cribriform plate were far less commonly the location of CSF fistulas than the cribriform plate and specifically the posterior third of the cribriform plate.

A possible explanation is that the cribriform plate might be widest in its posterior aspect. Hence the area in which defects may form is comparatively larger and thus the incidence of leaks in this region is higher. Moreover the anterior cribriform plate is strongly buttressed by the thick bone of the crista galli. Given the thinness of the bone and its multiple perforations from the olfactory filia, the lack of lateral buttressing and the increased surface area in the posterior cribriform plate creates a point of maximum weakness and highest predisposition to CSF fistula formation in the face of chronically increased ICP.

Surgical approaches for the repair of anterior cranial fossa leaks traditionally were carried out via a frontal transcranial approach. Although this approach does have some advantages, such as the ability to place a large vascularized pericranial flap, it does also have significant risks, such as frontal lobe injury, frontal sinus damage, and the obliteration of the superior sagittal sinus.,14,15,18 Additionally, this is a relatively nonselective approach, by which wide access to the cranial surface of the skull base is gained, with the inner surface of the skull then blanketed with graft material in a nonselective fashion. Success rates for this approach were on the order of $80 \% .{ }^{9}$ With the advent of endoscopy as the surgical approach to the repair of CSF leaks of the anterior cranial base, given that no brain retraction is required and the approach trajectory avoids the frontal sinus and superior sagittal sinus, many of these complications can be avoided. Success rates are higher, on the order of $94 \%,{ }^{3,4,12,14}$ However, the blanketing approach that can be used in transcranial surgery is not applicable to endoscopic surgery which requires a much higher degree of anatomical localization.

Limitations to this study include the retrospective nature, which inherently incurs some element of recall bias; specifically, minor episodes of cranial trauma might have been forgotten or not thought relevant. Moreover, operative reports dictated at the time of surgery in some cases did not contain enough intraoperative detail to definitively categorize anatomical location of defects according to the grid used in this study. The effect of this limitation should not have an impact on the validity of this study, because we excluded all cases in which preoperative imaging studies were not available for confirming lesion location and obtaining the required measurements. Additionally, the assumption that the epicenter of a current defect represents the origin of the defect might be incorrect. Bony remodeling and areas of increased structural strength may lead to displacement of structures to the point where anatomy becomes warped and the current epicenter is spatially remote from the point of origin. The surface area represented by individual elements within our grid is not equal. Anatomically, the nasal roof is wider anteriorly than posteriorly, and this was normalized, so as to superimpose the fistula locations on a $40 \mathrm{~mm} \times 40-\mathrm{mm}$ grid. This leads to an overrepresentation of the anterior aspects within the final grid, although it did not seem to introduce significant error, given the preponderance of lesions within the posterior aspect of the cribriform plate. Spatial warping inherent in the normalization process precludes the possibility of performing a direct comparison of the size of the defects. Lastly, although the statistical analysis of the data of this study does demonstrate a nonrandom distribution, the study is limited by the relatively small size of the study population.

\section{Conclusions}

Our findings suggest that spontaneous CSF leaks are nonrandomly distributed along the anterior cranial fossa. The cribriform plate is the most likely site of leakage, with a trend toward the posterior third being more often involved than the anterior and middle third. Clinicians searching for occult spontaneous leaks of the anterior skull base should examine the cribriform plate, especially 
the posterior third, with particularly close scrutiny. The most likely explanation for our finding is that the width of the cribriform plate increases posteriorly, thereby increasing the surface area of thin bone. Moreover, the absence of the crista galli decreases the lateral buttressing of the posterior cribriform plate.

\section{References}

1. Aarabi B, Leibrock LG: Neurosurgical approaches to cerebrospinal fluid rhinorrhea. Ear Nose Throat J 71:300-305, 1992

2. Alonso RC, de la Peña MJ, Caicoya AG, Rodriguez MR, Moreno EA, de Vega Fernandez VM: Spontaneous skull base meningoencephaloceles and cerebrospinal fluid fistulas. Radiographics 33:553-570, 2013

3. Banu MA, Kim JH, Shin BJ, Woodworth GF, Anand VK, Schwartz TH: Low-dose intrathecal fluorescein and etiologybased graft choice in endoscopic endonasal closure of CSF leaks. Clin Neurol Neurosurg 116:28-34, 2014

4. Bedrosian JC, Anand VK, Schwartz TH: The endoscopic endonasal approach to repair of iatrogenic and noniatrogenic cerebrospinal fluid leaks and encephaloceles of the anterior cranial fossa. World Neurosurg 82 (6 Suppl):S86-S94, 2014

5. Chaaban MR, Illing E, Riley KO, Woodworth BA: Spontaneous cerebrospinal fluid leak repair: a five-year prospective evaluation. Laryngoscope 124:70-75, 2014

6. Dare AO, Balos LL, Grand W: Neural-dural transition at the medial anterior cranial base: an anatomical and histological study with clinical applications. J Neurosurg 99:362-365, 2003

7. Daudia A, Biswas D, Jones NS: Risk of meningitis with cerebrospinal fluid rhinorrhea. Ann Otol Rhinol Laryngol 116:902-905, 2007

8. Erdem G, Erdem T, Miman MC, Ozturan O: A radiological anatomic study of the cribriform plate compared with constant structures. Rhinology 42:225-229, 2004

9. Hubbard JL, McDonald TJ, Pearson BW, Laws ER Jr: Spontaneous cerebrospinal fluid rhinorrhea: evolving concepts in diagnosis and surgical management based on the Mayo Clinic experience from 1970 through 1981. Neurosurgery 16:314-321, 1985

10. Kainz J, Stammberger H: [The roof of the anterior ethmoid: a locus minoris resistentiae in the skull base.] Laryngol Rhinol Otol (Stuttg) 67:142-149, 1988 (Ger)

11. Keros P: [On the practical value of differences in the level of the lamina cribrosa of the ethmoid.] Z Laryngol Rhinol Otol 41:809-813, 1962 (Ger)

12. Komotar RJ, Starke RM, Raper DMS, Anand VK, Schwartz TH: Endoscopic endonasal versus open repair of anterior skull base CSF leak, meningocele, and encephalocele: a systematic review of outcomes. J Neurol Surg A Cent Eur Neurosurg 74:239-250, 2013

13. Lewin W: Cerebrospinal fluid rhinorrhoea in closed head injuries. Br J Surg 42:1-18, 1954
14. Nyquist GG, Anand VK, Mehra S, Kacker A, Schwartz TH: Endoscopic endonasal repair of anterior skull base non-traumatic cerebrospinal fluid leaks, meningoceles, and encephaloceles. J Neurosurg 113:961-966, 2010

15. Park JI, Strelzow VV, Friedman WH: Current management of cerebrospinal fluid rhinorrhea. Laryngoscope 93:12941300,1983

16. Patron V, Berkaoui J, Jankowski R, Lechapt-Zalcman E, Moreau S, Hitier M: The forgotten foramina: a study of the anterior cribriform plate. Surg Radiol Anat 37:835-840, 2015

17. Psaltis AJ, Overton LJ, Thomas WW III, Fox NF, Banks CA, Schlosser RJ: Differences in skull base thickness in patients with spontaneous cerebrospinal fluid leaks. Am J Rhinol Allergy 28:e73-e79, 2014

18. Ray BS, Bergland RM: Cerebrospinal fluid fistula: clinical aspects, techniques of localization, and methods of closure. $\mathbf{J}$ Neurosurg 30:399-405, 1969

19. Rouvière H: Précis d'anatomie et de dissection. Paris: Masson, 1911

20. Schlosser RJ, Bolger WE: Nasal cerebrospinal fluid leaks: critical review and surgical considerations. Laryngoscope 114:255-265, 2004

21. Schlosser RJ, Bolger WE: Significance of empty sella in cerebrospinal fluid leaks. Otolaryngol Head Neck Surg 128:32-38, 2003

22. Schlosser RJ, Bolger WE: Spontaneous nasal cerebrospinal fluid leaks and empty sella syndrome: a clinical association. Am J Rhinol 17:91-96, 2003

23. Woodworth BA, Prince A, Chiu AG, Cohen NA, Schlosser RJ, Bolger WE, et al: Spontaneous CSF leaks: a paradigm for definitive repair and management of intracranial hypertension. Otolaryngol Head Neck Surg 138:715-720, 2008

\section{Disclosures}

The authors report no conflict of interest concerning the materials or methods used in this study or the findings specified in this paper.

\section{Author Contributions}

Conception and design: Schwartz, Murray, Anand. Acquisition of data: Murray, Friedlander, Hanz, Singh. Analysis and interpretation of data: Murray. Drafting the article: Murray. Critically revising the article: Schwartz, Murray. Reviewed submitted version of manuscript: Schwartz, Murray. Approved the final version of the manuscript on behalf of all authors: Schwartz. Statistical analysis: Murray. Administrative/technical/material support: Murray.

\section{Correspondence}

Theodore H. Schwartz, Department of Neurosurgery, Weill Cornell Medical College, NewYork-Presbyterian Hospital, 525 East 68th St., Box 99, New York, NY 10065. email: schwarh@med. cornell.edu. 